\title{
Microduplication of 7q36.3 encompassing the SHH long-range regulator (ZRS) in a patient with triphalangeal thumb-polysyndactyly syndrome and congenital heart disease
}

\author{
ZHENGHUA LIU $^{1}$, NI YIN ${ }^{1}$, LIANGHUI GONG ${ }^{1}$, ZHIPING TAN $^{1,2}$, \\ BANGLIANG YIN $^{1}$, YIFENG YANG ${ }^{1,2}$ and CHENG LUO ${ }^{1}$ \\ ${ }^{1}$ Department of Cardiothoracic Surgery; ${ }^{2}$ Clinical Center for Gene Diagnosis and Therapy of State Key Laboratory of \\ Medical Genetics, The Second Xiangya Hospital, Central South University, Changsha, Hunan 410011, P.R. China
}

Received November 21, 2015; Accepted November 26, 2016

DOI: $10.3892 / \mathrm{mmr} .2016 .6092$

\begin{abstract}
Triphalangeal thumb-polysyndactyly syndrome (TPT-PS) is an autosomal dominant disorder with complete penetrance and a variable expression consisting of opposable triphalangeal thumbs, duplication of the distal thumb phalanx, pre-axial polydactyly and duplication of the big toes (hallux). The causative gene of TPT-PS has been mapped to 7q36.3. Sonic hedgehog (SHH) expressed in the zone of polarizing activity (ZPA) has an important role in defining the anterior-posterior axis and numbers of digits in limb bud development. Point mutation or duplication in the ZPA regulatory sequence (ZRS), a cis-regulator of SHH, will lead to TPT-PS. The present study describes a 1-year-old female congenital heart disease (CHD) patient with TPT-PS phenotype. In this Han Chinese family with TPT-PS, high resolution single nucleotide polymorphism array technology identified a novel $0.29 \mathrm{Mb}$ duplication comprising ZRS at 7q36.3 where $L M B R 1$ is located. Additionally, a novel deletion of 22q11.21 was detected in the proband with Tetralogy of Fallot. However, the parents and other relatives of the patient did not harbor this genomic lesion nor CHD. The findings supported the hypothesis that an increased copy number variation of ZRS is the genetic mechanism underlying the phenotype of TPT-PS, and corroborated that 22q11.21 deletion is a genetic cause of CHD.
\end{abstract}

\section{Introduction}

Triphalangeal thumb-polysyndactyly syndrome (TPT-PS; OMIM \#174500), also called pre-axial polydactyly type II (PPD2; OMIM \#174500), is a well-defined autosomal dominant

Correspondence to: Dr Cheng Luo, Department of Cardiothoracic Surgery, The Second Xiangya Hospital, Central South University, 139 Renmin Road, Changsha, Hunan 410011, P.R. China

E-mail: chengluo_lolo@hotmail.com

Key words: triphalangeal thumb-polysyndactyly syndrome, 7q36.3 duplication, ZRS, 22q11.21 deletion, SNP-array, CNV, congenital heart disease, Tetralogy of Fallot disorder with complete penetrance and variable expression. The phenotype consists of opposable triphalangeal thumbs, duplication of distal thumb phalanx, pre-axial polydactyly, and duplication of the big toes (hallux).

Using linkage analysis, a malformation linked, highly polymorphic locus was identified within a large Dutch family with TPT-PS and, for the first time, the disease locus was located at the 7q36 region (1). Variable expression of TPT-PS was demonstrated by the asymmetrical limb deformities of affected individuals and the differences observed between monozygotic twins in that family.

The Sonic hedgehog (SHH) protein secreted by the mesenchymal cells is restricted to a region termed the zone of polarizing activity (ZPA) in the limb bud as a key regulator in defining the anterior-posterior axis and numbers of digits in early limb development and morphogenesis $(2,3)$. In 2002, a translocation breakpoint was first identified in a pre-axial polydactyly patient (4). In 2003, it was reported that point mutations segregated with polydactyly in three unrelated families with PPD2 and in the Hemimelic extra toes mouse mutant. A further study identified a cis-regulator, designated ZPA regulatory sequence (ZRS), driving normal SHH expression in ZPA, which lies at $1 \mathrm{Mb}$ upstream of the target gene $S H H$ within intron 5 of the Limb region 1 homolog (LMBRI; OMIM \#605522) (5). The ZRS is highly conserved from mammals to fish and can drive the expression of a reporter gene in ZPA $(5,6)$. Subsequently, several different point mutations that are clustered within a highly conserved region of ZRS in humans have been reported to be associated with TPT-PS (7-9).

In 2008, for the first time in humans, a 588,819 bp duplication of 7q36 comprising ZRS was reported in a large family with TPT-PS (10). This previous study demonstrated that a duplication of ZRS results in a similar phenotype, that is, TPT-PS as single nucleotide mutations within ZRS. In the same year, six duplications of at least 131, 291, 158, 246, 235 and $398 \mathrm{~kb}$ were identified in six families with TPT-PS (11). These duplications shared a 32,757 bp common overlapping segment containing the ZRS enhancer.

A previous study (12) suggested that copy number variations (CNVs) can be associated with syndromic congenital 
heart disease (CHD), including limb anomalies. The present study reported the identification of a novel $0.29 \mathrm{Mb}$ duplication in 7q36.3 encompassing ZRS in LMBR 1 in a 1-year-old female patient with the TPT-PS phenotype and CHD associated with a de novo 22q11.21 deletion.

\section{Materials and methods}

Ethical approval and patient consent. The present study was approved by the Review Board of The Second Xiangya Hospital of the Central South University (Changsha, China). All individuals provided informed written consent. Blood samples were collected following informed written consent from the patient's parent on behalf of the child enrolled.

Clinical presentation. The present study investigated a four-generation family from Central-South China (Hunan) with a phenotype comprising triphalangeal thumbs and pre-axial polysyndactyly leading to the diagnosis of TPT-PS, and the phenotype varied among affected individuals. The proband, a 1-year-old female patient, was the second child of non-consanguineous parents. Central cyanosis, cardiac murmur and a malformation of both thumbs were observed at birth.

At 1 year of age, the patient was referred to the in-patient department of The Second Xiangya Hospital of the Central South University (Changsha, Hunan, China) for surgical treatment of cardiac defects. Tetralogy of Fallot (TOF) was diagnosed by two-dimensional color Doppler echocardiography. Physical examination highlighted the limb anomalies of the proband, which comprised triphalangeal thumbs of both hands and polysyndactyly in the right thumb, and so typical TPT-PS was diagnosed (Fig. 1). Next, the patient's father was also ascertained to be expressing triphalangeal thumbs bilaterally but without polysyndactyly, and CHD was excluded using color Doppler echocardiography. A pedigree analysis revealed that the proband's grandmother, one of the grandmother's brothers and the grandmother's mother manifested similar thumb phenotypes, but had all died a number of years previously. This indicated that the phenotype was transmitted in an autosomal dominant manner throughout four generations in the family (Fig. 2). The pedigree had no history of CHD, with the exception of the female patient with TOF.

Cytogenetic analysis. Chromosome analysis was performed on the peripheral blood samples of the patient and the patient's parents by conventional G-Banded techniques (550 bands resolution). A $2 \mathrm{ml}$ sample of peripheral blood was collected from each individual. All samples were subjected to lymphocyte culture according to standard cytogenetic protocol.

DNA extraction. Genomic DNA was prepared from peripheral blood using a DNeasy Blood and Tissue kit (Qiagen, Inc., Valencia, CA, USA) on the QIAcube automated DNA extraction robot (Qiagen GmbH, Hilden, Germany) following the manufacturer's protocol.

Sequence analysis. The entire coding regions, including the flanking intronic sequences of LMBRI (RefSeq: NM_022458) and TBXI (RefSeq: NM_080647) were amplified with polymerase chain reaction (PCR; primer sequences are presented in Table I and the supplier was Sangon Biotech Co., Ltd., Shanghai, China). Sequences of the PCR products were determined using the ABI 3100 Genetic Analyzer (Applied Biosystems; Thermo Fisher Scientific, Inc., Waltham, MA, USA), as previously described (13).

Single nucleotide polymorphism (SNP) array analysis. Genomic DNA samples were adjusted to a final concentration of $50 \mathrm{ng} / \mathrm{ml}$. The Human Omni1-Quad Chip (Illumina, Inc., San Diego, CA, USA) and the Illumina BeadScan genotyping system (Beadstation Scanner) were employed to obtain the signal intensities of SNP probes. The Genome Studio software (version 2011; Illumina, Inc.) was used to analyze the genotypes [human genome build 37 (Hg19)] and to evaluate the experimental quality. The call rates of the samples were $>99.0 \%$.

\section{Results}

The karyotypes of the patient and the patient's parents were normal. Sequence analysis did not identify any missense or nonsense mutation in LMBRI and TBXI. To explore the presence of genomic imbalances, an SNP-array system was employed to analyze the whole genome for CNVs (Human Omnil-Quad Chip, Beadstation Scanner and GenomeStudio V2011 software). A total of $343 \mathrm{CNVs}$ were identified. By comparing these CNVs with the Database of Genomic Variants (http://dgv.tcag.ca), the present study identified a novel $0.29 \mathrm{Mb}$ duplication of chromosome 7q36.3 (Chr7: 156,484,201-156,772,643), which contains two genes, LMBRI and NOM1 (Fig. 3). The patient's TPT-PS affected father also harbored the same duplication, however, the patient's TPT-PS unaffected mother did not.

In addition, the present study also identified a de novo 2.6 Mb deletion of chromosome 22q11.21 (chr22: $18,874,965-21,464,479)$ in the proband. This $2.6 \mathrm{Mb}$ deletion region includes TBXI, HIRA, RTN4R and numerous other genes (Fig. 4). However, the proband's parents did not harbor the 22q11.21 deletion.

\section{Discussion}

The present study reported a complication that multiple pathogenic CNVs co-exist in one patient, and consequently resulted in congenital multi-system diseases, complicating limb anomalies with congenital heart defects.

The duplicated region of chromosome $7 \mathrm{q} 36.3$ in the patient reported in the present study spans 288,443 bp containing the genes $L M B R 1$ and, in part, NOM1. To the best of our knowledge, this is a novel 7q36.3 duplication. The duplication was identified to co-segregate with the TPT-PS phenotype in the patient and the patient's father, but was not present in the family members unaffected by TPT-PS. This result notably suggested that the duplication involving ZRS is the disease-causing mutation underlying human TPT-PS.

Intron 5 of $L M B R 1$ contains a cis-acting regulator of limb-specific SHH, which is designated as ZRS $(2,5)$. The role of ZRS in limb development has been interpreted as an enhancer driving SHH expression in the posterior limb bud, as well as a repressor that silences the anterior expression. The direct link between ZRS and the PPD phenotypes was 
Table I. Primer sequences used to amplify ZRS (on LMBR1) and TBX1.

\begin{tabular}{llll}
\hline Gene & Exon & \multicolumn{1}{c}{ Forward primer (5'-3') } & \multicolumn{1}{c}{ Reverse primer (5'-3') } \\
\hline LMBR1 & ZRS-1 & TTTCAAATGCTCACTTTACATGG & TTTTATGACCAGATGACTTTTTCC \\
ZBX1 & AGS-2 & AGCCAGTGCGTTCAGCATCGCCTC & AAGAGCTGCCTCCACCTACTTTG \\
& $3-1$ & TGATCTCCGCCGTGTCCAGC & GGCCACCTTCGCGTTCTTCTT \\
& $3-2$ & GGTGAAGAAGAACGCGAAGGTGG & GACGGCGAACAGCGAAGGAG \\
& TGCCTTCCACCAGCTAGG & AGACGACCCTTGGAGTTGG \\
& 5 & CTCTGGGTTCACCTCCACAT & CAGGCCTCTTAGGGACAGG \\
& 6 & CTCCCACCCCAGATCCTC & AATCCGCTCAGGTCCTCC \\
& 7 & TTGGTGCGCTTCTCCTAACACTC & AAGGCGCTCATGAGCGGCAGTG \\
& $9 \mathrm{~A}-1$ & GGGACTGTGACCCTGAGGACTG & TCAGCATTCAACAAAGACGG \\
$9 \mathrm{~A}-2$ & TCAGACACTGGACATTTGTGC & ACTGGGAGTGTGACTCTATGGA \\
& CAGAGTGCCAACCCTTCAAT & TGGAGGATTCGCTTCCATCACAG \\
& $9 \mathrm{~B}-1$ & AACACTTTGACCTTCCTCCACCC & CCCGAGATAGTGGTCGTAGGC \\
$9 \mathrm{C}-2$ & TCGCATGGGGCGTCGGAGCT & GCCGACGAGTACATGTTGGC \\
$9 \mathrm{C}-3$ & GGTGCTAAGCCCCTCGCTG & TATTCCTTGCTTGCCCTTGG
\end{tabular}

ZRS, ZPA regulatory sequence; $L M B R 1$, limb region 1 homolog; TBX1, T-box-1.

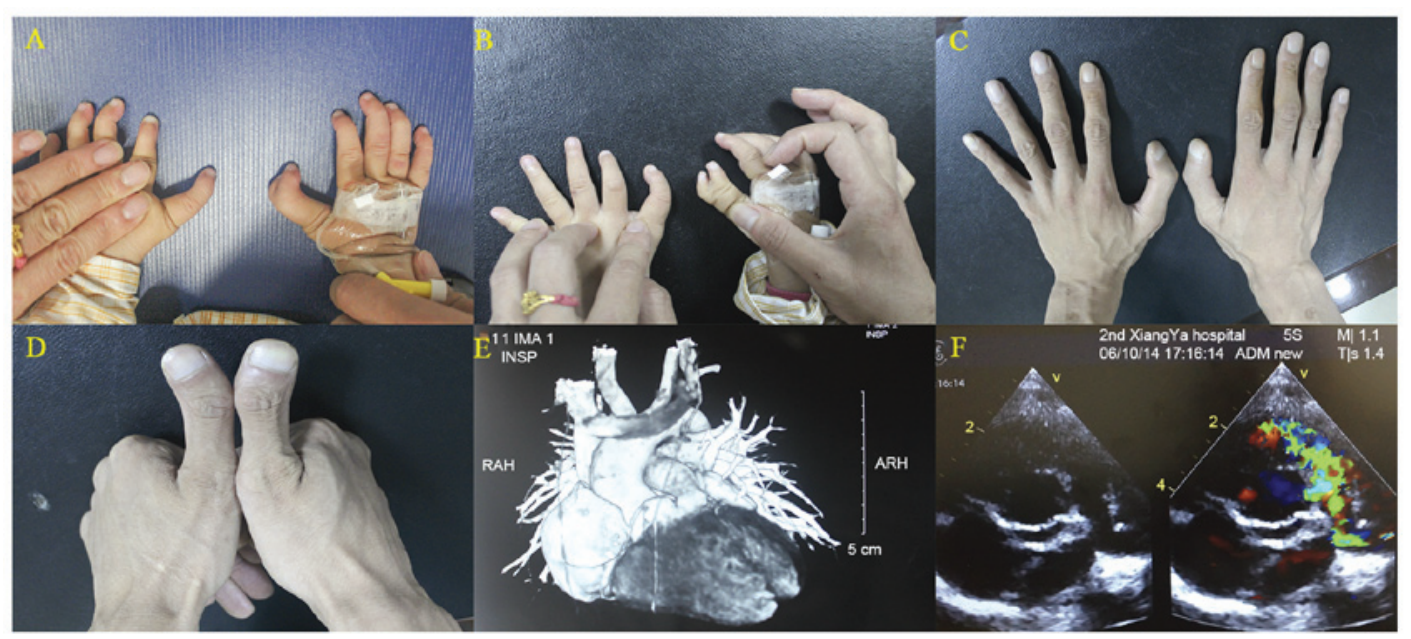

Figure 1. Clinical features. (A, B) The patient has triphalangeal thumbs on both hands and polysyndactyly in right thumb. (C, D) Her father has triphalangeal thumbs of both hands. (E) Computed tomography angiography and (F) echocardiogram of the patient indicate the Tetralogy of Fallot.

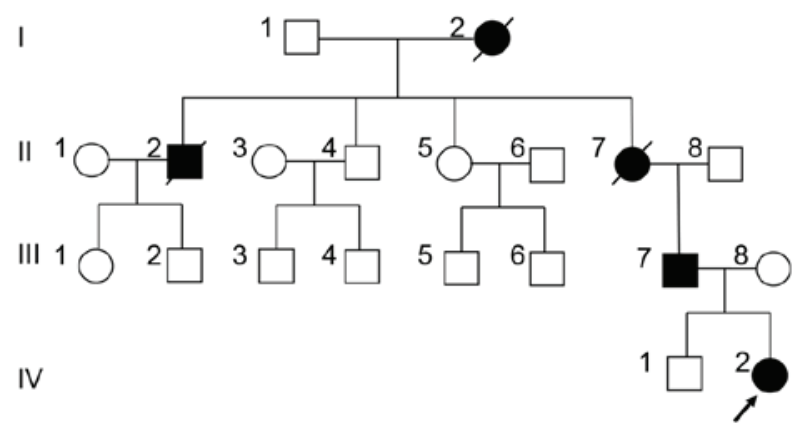

Figure 2. Pedigree of the triphalangeal thumb-polysyndactyly syndrome family. Squares indicate male members, circles, female; a symbol with a slash, the deceased members; closed symbols, the affected members; open symbols, unaffected members. The arrow indicates the proband, who presents with Tetralogy of Fallot as well. established by the identification of a number of the different point mutations within ZRS, including in polydactylous cats, in mouse models of PPD, and in human PPD families $(5-7,14)$. Duplication has been shown to be a major type of CNV in numerous human genetic diseases (15). One possible explanation is a gain of function mechanism leading to an augmented $\mathrm{SHH}$ expression in the limb bud. The pathogenicity of the duplication involving ZRS may be further supported by a previous study (16) in the Sasquatch mutant mouse whose phenotype is an isolated pre-axial polydactylous. This mutant was generated by the transgenic insertion of a reporter gene in intron 5 of Lmbrl and has been shown to have a $24 \mathrm{~kb}$ intronic duplication containing the mouse ZRS (5). In 2008, Klopocki et al (10) first reported the identification of a genomic 


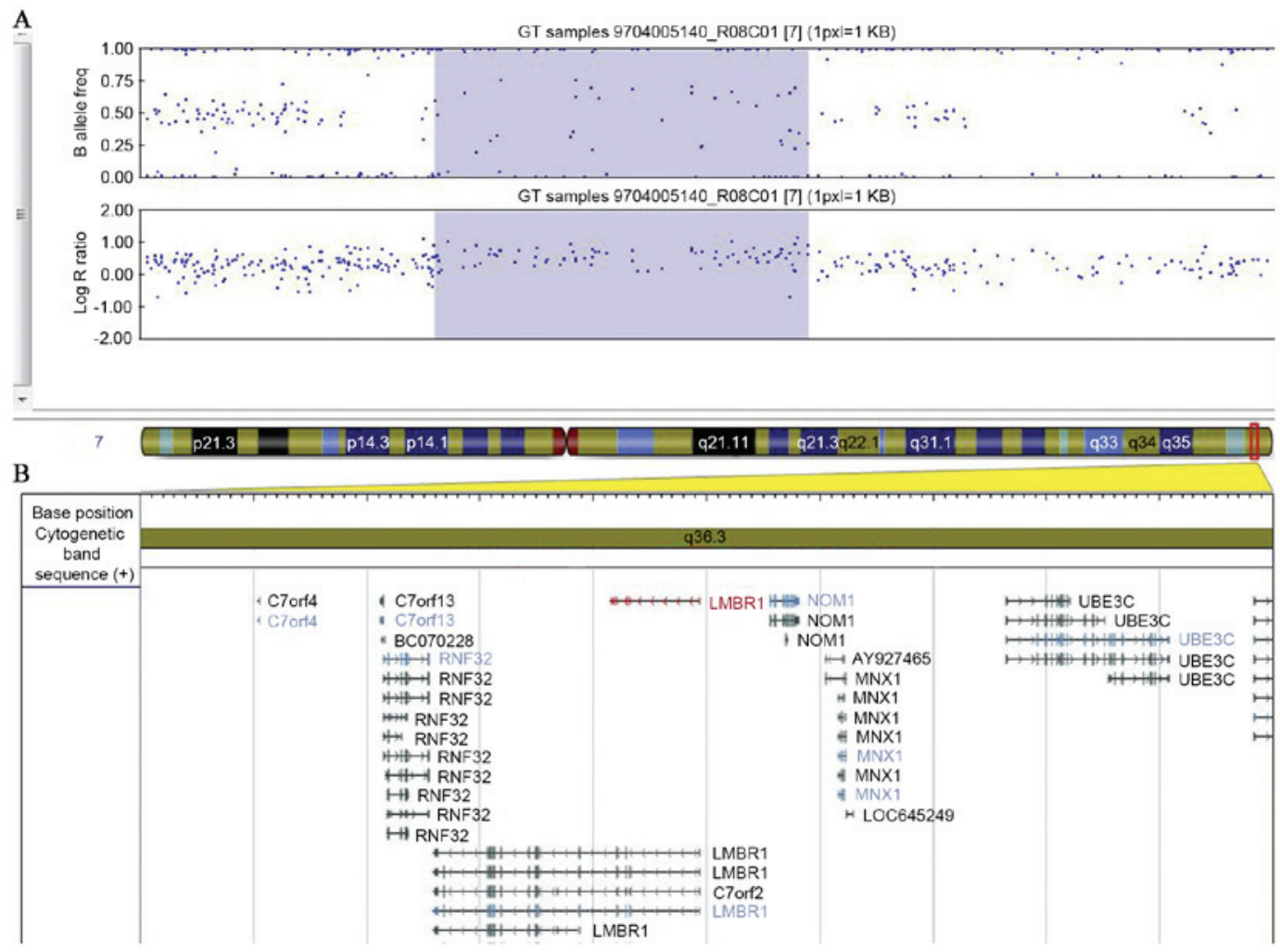

Figure 3. Illumina single nucleotide polymorphism-array result of the 7q36.3 region in proband. (A) A 288,443 bp duplication in 7q36.3 (Chr7: 156,484,201-156,772,643) (UCSC Genome Browser on Human GRCh37/hg19 Assembly). (B) Genes mapped to the duplicated region. GT, genotype.

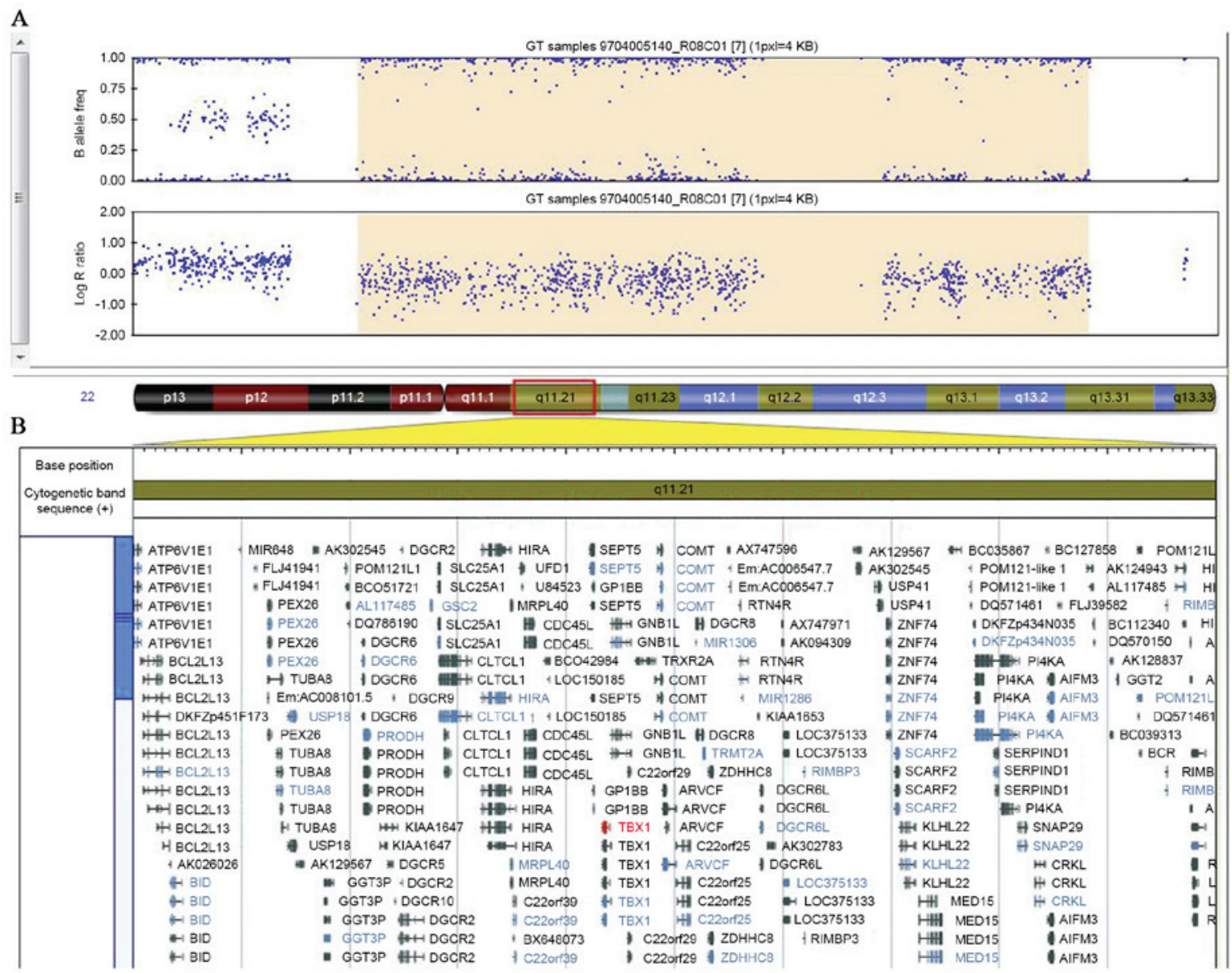

Figure 4. Illumina single nucleotide polymorphism-array result of the 22q11.21 region in proband. (A) A 2,589,515 bp deletion in $22 \mathrm{q} 11.21$ (chr22: 18,874,965-21,464,479) (UCSC Genome Browser on Human GRCh37/hg19 Assembly). (B) Genes mapped to the deletion region. GT, genotype. 
duplication containing ZRS in a large German family with TPT-PS. In the same year, Sun et al (11) identified six duplications in six families with TPT-PS. These independent findings and the results of the present study consistently implied that the duplication of ZRS long range enhancer is the molecular pathogenesis of TPT-PS. It is hypothesized that disruption of cis-regulator via ZRS duplication results in the dysregulation of SHH, leading to TPT-PS phenotypes.

The deletion of chromosome $22 \mathrm{q} 11.21$ in the patient reported in the present study spans $2,589,515 \mathrm{bp}$, containing TBX1, HIRA, RTN4R and numerous other genes. Chromosome 22 q11.2 deletion syndrome (DiGeorge syndrome; OMIM \#188400) is one of the most common genetic syndromes, with a prevalence of $1: 4,000$ to $1: 6,000(17,18)$. At least 30 genes have been mapped to the deleted region, among them $T B X 1$, a major genetic determinant of the 22q11.2 deletion syndrome (19). The phenotypic spectrum is varied, including CHD, velopharyngeal insufficiency and cleft palate facial anomalies, immune disorders, and hypocalcemia secondary to hypoparathyroidism (20). The most common cardiac defects are conotruncal anomalies, including TOF, TOF with pulmonary atresia, truncus arteriosus and interrupted aortic arch (21). In the present study, the patient was diagnosed as TOF, but presented neither obvious facial anomalies nor immune deficiencies. Her parents did not harbor the 22q11.21 deletion and did not have CHD. It is inferred that it was the patient's deletion of 22q11.21 that caused the CHD and TOF. This is the first report, to the best of our knowledge, on a co-existence of 7q36.3 duplication with 22q11.21 deletion in a syndromic CHD.

In conclusion, the present study described a duplication of 7q36.3 containing the ZRS long range enhancer of SHH in a family with distinctive phenotypic features of TPT-PS. Additionally, a de novo 22q11.21 deletion containing TBX1 was detected in the proband who is the unique member of the family with TOF, a type of CHD. The findings of the present study indicated that 7q36.3 duplication encompassing ZRS region is the underlying genetic cause of TPT-PS, and in addition, corroborate that $22 \mathrm{q} 11.21$ deletion is a genetic cause of CHD.

\section{Acknowledgements}

The present study was supported by the National Natural Science Foundation of China (no. 8140020813 to Cheng Luo). The authors would like to thank the patient and her parents for participating in this study. The authors are also grateful to the State Key Laboratory of Medical Genetics of China, in particular to Mr Qian Pan and Miss Yi-Qiao Hu, for technical support.

\section{References}

1. Tsukurov O, Boehmer A, Flynn J, Nicolai JP, Hamel BC, Traill S, Zaleske D, Mankin HJ, Yeon H and Ho C: A complex bilateral polysyndactyly disease locus maps to chromosome 7q36. Nat Genet 6: 282-286, 1994

2. Hill RE: How to make a zone of polarizing activity: Insights into limb development via the abnormality preaxial polydactyly. Dev Growth Differ 49: 439-448, 2007.

3. Radhakrishna U, Blouin JL, Solanki JV, Dhoriani GM and Antonarakis SE: An autosomal dominant triphalangeal thumb: Polysyndactyly syndrome with variable expression in a large Indian family maps to 7q36. Am J Med Genet 66: 209-215, 1996.
4. Lettice LA, Horikoshi T, Heaney SJ, van Baren MJ, van der Linde HC, Breedveld GJ, Joosse M, Akarsu N, Oostra BA, Endo N, et al: Disruption of a long-range cis-acting regulator for Shh causes preaxial polydactyly. Proc Natl Acad Sci USA 99: 7548-7553, 2002.

5. Lettice LA, Heaney SJ, Purdie LA, Li L, de Beer P, Oostra BA, Goode D, Elgar G, Hill RE and de Graaff E: A long-range Shh enhancer regulates expression in the developing limb and fin and is associated with preaxial polydactyly. Hum Mol Genet 12: 1725-1735, 2003.

6. Sagai T, Masuya H, Tamura M, Shimizu K, Yada Y, Wakana S, Gondo Y, Noda T and Shiroishi T: Phylogenetic conservation of a limb-specific, cis-acting regulator of Sonic hedgehog (Shh). Mamm Genome 15: 23-34, 2004.

7. Gurnett CA, Bowcock AM, Dietz FR, Morcuende JA, Murray JC and Dobbs MB: Two novel point mutations in the long-range SHH enhancer in three families with triphalangeal thumb and preaxial polydactyly. Am J Med Genet A 143A: 27-32, 2007.

8. Wang ZQ, Tian SH, Shi YZ, Zhou PT, Wang ZY, Shu RZ, Hu L and Kong X: A single $C$ to $T$ transition in intron 5 of LMBR 1 gene is associated with triphalangeal thumb-polysyndactyly syndrome in a Chinese family. Biochem Biophys Res Commun 355: 312-317, 2007.

9. Al-Qattan MM, Al Abdulkareem I, Al Haidan Y and Al Balwi M: A novel mutation in the SHH long-range regulator (ZRS) is associated with preaxial polydactyly, triphalangeal thumb, and severe radial ray deficiency. Am J Med Genet A 158A: 2610-2615, 2012.

10. Klopocki E, Ott CE, Benatar N, Ullmann R, Mundlos S and Lehmann K: A microduplication of the long range SHH limb regulator (ZRS) is associated with triphalangeal thumb-polysyndactyly syndrome. J Med Genet 45: 370-375, 2008.

11. Sun M, Ma F, Zeng X, Liu Q, Zhao XL, Wu FX, Wu GP, Zhang ZF, $\mathrm{Gu} \mathrm{B}$, Zhao YF, et al: Triphalangeal thumb-polysyndactyly syndrome and syndactyly type IV are caused by genomic duplications involving the long range, limb-specific SHH enhancer. J Med Genet 45: 589-595, 2008.

12. Luo C, Yang YF, Yin BL, Chen JL, Huang C, Zhang WZ, Wang J, Zhang H, Yang JF and Tan ZP: Microduplication of 3p25.2 encompassing RAF1 associated with congenital heart disease suggestive of Noonan syndrome. Am J Med Genet A 158A: 1918-1923, 2012

13. Tan ZP, Huang $\mathrm{C}, \mathrm{Xu} \mathrm{ZB}$, Yang JF and Yang YF: Novel ZFPM2/FOG2 variants in patients with double outlet right ventricle. Clin Genet 82: 466-471, 2012.

14. Masuya H, Sezutsu H, Sakuraba Y, Sagai T, Hosoya M, Kaneda H, Miura I, Kobayashi K, Sumiyama K, Shimizu A, et al: A series of ENU-induced single-base substitutions in a long-range cis-element altering Sonic hedgehog expression in the developing mouse limb bud. Genomics 89: 207-214, 2007.

15. Beckmann JS, Estivill X and Antonarakis SE: Copy number variants and genetic traits: Closer to the resolution of phenotypic to genotypic variability. Nat Rev Genet 8: 639-646, 2007.

16. Sharpe J, Lettice L, Hecksher-Sorensen J, Fox M, Hill R and Krumlauf R: Identification of sonic hedgehog as a candidate gene responsible for the polydactylous mouse mutant Sasquatch. Curr Biol 9: 97-100, 1999.

17. Goodship J, Cross I, LiLing J and Wren C: A population study of chromosome 22q11 deletions in infancy. Arch Dis Child 79: 348-351, 1998 .

18. Botto LD, May K, Fernhoff PM, Correa A, Coleman K, Rasmussen SA, Merritt RK, O'Leary LA, Wong LY, Elixson EM, et al: A population-based study of the 22q11.2 deletion: Phenotype, incidence, and contribution to major birth defects in the population. Pediatrics 112: 101-107, 2003.

19. Yagi H, Furutani Y, Hamada H, Sasaki T, Asakawa S, Minoshima S, Ichida F, Joo K, Kimura M, Imamura S, et al: Role of TBX1 in human del22q11.2 syndrome. Lancet 362: 1366-1373, 2003.

20. Shprintzen RJ: Velo-cardio-facial syndrome: 30 Years of study. Dev Disabil Res Rev 14: 3-10, 2008.

21. Momma K: Cardiovascular anomalies associated with chromosome 22q11.2 deletion syndrome. Am J Cardiol 105: 1617-1624, 2010 . 